Revista Brasileira de Meteorologia, v.29, n.2, 307 - 313, 2014

\title{
ESTIMATIVA DA TEMPERATURA BASAL INFERIOR PARA AS CULTIVARES DE OLIVEIRA GRAPPOLO E MARIA DA FÉ
}

\author{
PLÍNIO MARCOS BERNARDO DE SOUZA E FABRINA BOLZAN MARTINS \\ Universidade Federal de Itajubá, Itajubá, MG, Brasil \\ pliniobsouza@yahoo.com.br, fabrinabm@gmail.com \\ Recebido Junho de 2013 - Aceito Agosto de 2013
}

\begin{abstract}
RESUMO
A temperatura basal inferior $(\mathrm{Tb})$ é uma variável de entrada muito utilizada em modelos que quantificam o desenvolvimento de culturas agrícolas e florestais. O objetivo deste trabalho foi estimar a $\mathrm{Tb}$ para o desenvolvimento vegetativo na fase de muda, para duas cultivares de oliveira, Grappolo e Maria da Fé, em condições de campo. O estudo foi desenvolvido na área Experimental da Empresa de Pesquisa Agropecuária de Minas Gerais, em Maria da Fé, MG, Brasil, conduzido sob o delineamento inteiramente casualizado, sendo duas cultivares de oliveira, três épocas de transplantio, e quinze repetições. A Tb foi estimada através de seis métodos, cujos valores obtidos variaram em função do método de cálculo utilizado. A Tb estimada foi de $9,6^{\circ} \mathrm{C}$ para a Grappolo e $6,9^{\circ} \mathrm{C}$ para a Maria da Fé. Palavras-chave: Desenvolvimento, Temperatura do ar, Fenologia, Olea europaea L.
\end{abstract}

\begin{abstract}
ESTIMATE OF THE BASE TEMPERATURE IN TWO OLIVE CULTIVARS: GRAPPOLO AND MARIA DA FÉ

The base temperature $(\mathrm{Tb})$ is an input variable widely used in models of agricultural and forest crops development. The aim of this study was to estimate the $\mathrm{Tb}$ for vegetative development, represented by seedling phase in two olive cultivars under field conditions. The experiment was carried out in the experimental area of the Agricultural Research Corporation of Minas Gerais, in Maria da Fé, Minas Gerais, Brazil. The experimental design was completely randomized with two olive cultivars, three transplanting dates and fifteen repetitions. The $\mathrm{Tb}$ was estimated by six methods described in literature, whose values varied depending on each method of calculation. The estimated $\mathrm{Tb}$ was $9.6^{\circ} \mathrm{C}$ for Grappolo and $6.9^{\circ} \mathrm{C}$ to Maria da Fé, respectively.
\end{abstract}

Keywords: Development, Air temperature, Phenology, Olea Europaea L.

\section{INTRODUÇÃO}

A temperatura do ar é considerada o principal elemento meteorológico que afeta o desenvolvimento vegetativo e reprodutivo da maioria das espécies vegetais (Arnold, 1959; Martins et al., 2012). O conhecimento das necessidades térmicas do desenvolvimento vegetativo (emissão de folhas) e do desenvolvimento reprodutivo (inflorescência, florescimento, frutificação e maturação) (Sanz-Cortéz et al., 2002), é vital para otimizar as estratégias de manejo, melhorar a produção e a qualidade de mudas, auxiliar em programas de seleção e melhoramento, e escolher as espécies ou cultivares mais adaptadas às condições climáticas do local de cultivo (Martins et al., 2012; Müller et al., 2009).
A maneira mais simples e frequentemente usada para descrever o efeito da temperatura do ar sobre o desenvolvimento vegetal é através dos graus-dia, cuja unidade é ${ }^{\circ} \mathrm{C}$ dia (Lima e Silva, 2008; Lago et al., 2009). Os graus-dia é baseado no acúmulo térmico diário dentro dos quais a planta se desenvolve, sendo delimitados pelas temperaturas basais superior (TB) e inferior (Tb) de cada espécie (Lozada e Angelocci, 1999; Müller et al., 2009). Ambas temperaturas base (TB e Tb) representam, respectivamente, a temperatura acima e abaixo da qual o desenvolvimento é nulo ou desprezível para fins de cálculo. Geralmente, a TB assume valores elevados, os quais dificilmente são atingidos durante o ciclo de desenvolvimento no campo (Müller et al., 2009). Já a Tb assume valores baixos, os quais ocorrem principalmente durante o inverno, sendo bastante 
utilizada na clássica equação de cálculo dos graus-dia a qual é dada pela diferença entre a temperatura média do ar diária e a Tb da espécie (Arnold, 1959; Rosa et al., 2009). Assim, para uma estimativa precisa do desenvolvimento vegetal de uma determinada espécie, é necessário uma Tb adequada (Lago et al., 2009).

Na maioria dos casos, a Tb é determinada por métodos estatísticos com base em dados de observações fenológicas e de temperatura média do ar (Andrade, 2004; Lago et al., 2009). Existe uma variedade de métodos estatísticos utilizados para estimar a Tb, sendo os mais utilizados: desvio padrão em dias e graus-dia, coeficiente de variação em dias e graus-dia, o coeficiente de regressão, o X intercepto e o menor quadrado médio do erro (Arnold, 1959; Yang et al., 1995; Lozada e Angelocci, 1999; Sinclair et al., 2004; Lago et al., 2009). Esses métodos são uma ferramenta importante na estimativa da $\mathrm{Tb}$, porém, em virtude das aproximações existentes, poderão ocorrer diferenças entre a $\mathrm{Tb}$ fisiológica e a Tb estimada por tais métodos (Arnold, 1959; Yang et al., 1995; Lago et al., 2009). Além disso, a $\mathrm{Tb}$ possui diferença entre espécies, entre cultivares e ainda pode variar em função da fase de desenvolvimento que a planta se encontra (Lago et al., 2009). Desta maneira, deve haver cautela na utilização e interpretação dos valores de $\mathrm{Tb}$ obtidos estatisticamente, analisando o significado e os erros que podem conter (Andrade, 2004).

Estudos sobre a estimativa da Tb são amplamente realizados para culturas agrícolas como: ervilha (Barbano et al., 2002), soja (Sinclair et al., 2004), milho (Yang et al., 1995), feijão (Andrade, 2004), arroz (Lago et al., 2009), triticale (Pedro Jr. et al., 2004), melancia (Lucas et al., 2012), canola (Luz et al., 2012); culturas utilizadas para fins ornamentais e medicinais como aspília (Fagundes et al., 2010); e pouco realizados para culturas perenes, sendo alguns exemplos: café (Lima e Silva, 2008), eucalipto (Martins et al., 2007) e araucária (Assumpção Neto, 2008).

Estudos dessa natureza devem ser realizados, principalmente, em espécies potencialmente promissoras, no qual se deseja conhecer a adaptabilidade de cultivo fora do seu centro de origem, visando determinar as exigências bioclimáticas em cada fase do desenvolvimento (Ayerza e Sibbett, 2001; Luz et al., 2012). Uma espécie potencialmente promissora para os estados da região sul e sudeste do país é a oliveira (Olea europaea L.) (Vieira Neto et al., 2011), a qual é uma árvore frutífera subtropical de grande longevidade e de importância no cenário econômico mundial, com cultivos recentes e em expansão em vários estados brasileiros, principalmente em Minas Gerais (Lisboa et al., 2012).

Devido à escassez de estudos dessa natureza, o objetivo deste trabalho foi determinar a temperatura basal inferior para o desenvolvimento vegetativo em duas cultivares de oliveira,
Grappolo e Maria da Fé, durante a sua fase de muda em condições de campo.

\section{MATERIAL E MÉTODOS}

Foi conduzido um experimento a campo na área experimental da Empresa de Pesquisa Agropecuária de Minas Gerais (EPAMIG), Maria da Fé, MG, Brasil (latitude: $22^{\circ} 18^{\prime} 29^{\prime \prime}$ S; longitude: $45^{\circ} 22^{\prime} 31^{\prime \prime} \mathrm{W}$; e altitude: $1.276 \mathrm{~m}$ ). Segundo a classificação de Köppen, o clima do local é Cwb, subtropical de altitude com invernos secos e verões chuvosos e de temperaturas brandas (Vieira Neto et al., 2011).

O delineamento estatístico utilizado foi o inteiramente casualizado em esquema fatorial $2 \times 3$, sendo duas cultivares e três épocas de transplantio, com quinze repetições por tratamento. Cada repetição foi composta por mudas provenientes do enraizamento de estacas semilenhosas e ausentes de brotações. Utilizou-se a estaquia ao invés de sementes, como método de produção de mudas, devido ao longo processo de germinação de sementes de oliveira. As cultivares escolhidas foram 'Grappolo', destinada à produção de azeite e azeitona e, 'Maria da Fé', destinada à produção de azeite.

As épocas de transplantio foram instaladas a campo em intervalos de aproximadamente 30 dias, para que as plantas ficassem expostas a diferentes condições meteorológicas durante o seu desenvolvimento (Rosa et al., 2009). As datas de instalação foram: época 1 (E1) - 06/06/2011, época 2 (E2) - 08/07/2011, época 3 (E3) - 04/08/2011, as quais foram escolhidas por compreenderem os períodos em que as plantas estariam submetidas às menores temperaturas do ar, sendo possível estimar a Tb com maior precisão (Pedro Jr. et al. 2004; Sinclair et al., 2004).

Cada repetição foi plantada em vaso de polietileno de aproximadamente $4 \mathrm{~L}$, com espaçamento de $20 \mathrm{~cm}$ entre eles. Os vasos foram preenchidos com substrato constituído de terra e composto orgânico comercial (Provaso ${ }^{\circledR}$ ). Cada $\mathrm{m}^{3}$ de substrato foi enriquecido com $5 \mathrm{~kg}$ de superfosfato simples (18 dag kg-1 de $\left.\mathrm{P}_{2} \mathrm{O}_{5}\right), 1 \mathrm{~kg}$ de cloreto de potássio $\left(58 \mathrm{dag} \mathrm{\textrm {kg } ^ { - 1 }}\right.$ de $\left.\mathrm{K}_{2} \mathrm{O}\right)$ e $2,5 \mathrm{~kg}$ de calcário dolomítico (PRNT 100\%). Os vasos foram envolvidos com papel jornal visando minimizar a absorção da radiação solar e a consequente elevação da temperatura do substrato, o que poderia afetar a velocidade de desenvolvimento das mudas (Martins et al., 2007). Foi realizada irrigação regularmente com uso de regadores, de modo a minimizar o estresse hídrico nas plantas.

O desenvolvimento vegetativo foi quantificado pela variável número de folhas emitidas acumuladas (NF) pelas estacas de oliveira durante a fase de muda, as quais foram contadas uma vez por semana. O NF foi escolhido por ser 
considerado uma variável de desenvolvimento vegetativo, pois relaciona-se com a evolução da área foliar que influencia a interceptação da radiação solar (Rosa et al., 2009). A contagem das folhas foi iniciada quando havia uma folha visível, considerada como aquela com, no mínimo, $1,0 \mathrm{~cm}$ de comprimento (Sanz-Cortés et al., 2002) e finalizada quando cada repetição atingiu NF igual a 25 folhas, quando se considerou que a planta não mais se encontrava na fase de muda (Martins e Streck, 2007).

As variáveis meteorológicas, temperatura mínima e máxima diárias do ar, foram obtidas de uma estação meteorológica convencional pertencente ao Instituto Mineiro de Gestão das Águas (IGAM), localizada na EPAMIG à, aproximadamente, $50 \mathrm{~m}$ do local do experimento.

Para a estimativa da $\mathrm{Tb}$ é necessário calcular primeiramente os graus-dia (GD, ${ }^{\circ} \mathrm{C}$ dia), sendo dado pela expressão (Arnold, 1960):

$$
G D_{i}=\left(\frac{T M+T m}{2}\right)-T b
$$

em que: $\mathrm{GD}_{\mathrm{i}}$ é os graus-dia $\left({ }^{\circ} \mathrm{C}\right.$ dia), TM é a temperatura máxima diária do ar $\left({ }^{\circ} \mathrm{C}\right)$, Tm é a temperatura mínima diária do ar $\left({ }^{\circ} \mathrm{C}\right)$ e Tb é a temperatura basal inferior da cultivar. Para o cálculo do GD foi utilizada uma série de $\mathrm{Tb}$ variando de $0^{\circ}$ a $20^{\circ} \mathrm{C}$, em intervalos de $0,5^{\circ} \mathrm{C}$.

Os graus-dia acumulado $\left(\mathrm{GD},{ }^{\circ} \mathrm{C}\right.$ dia $)$ a partir da data do transplantio de cada época, foi obtida pelo somatório do $\mathrm{GD}_{\mathrm{i}}$ :

$$
G D=\sum G D_{i}
$$

Foram utilizadas as metodologias propostas por Arnold (1959) e Yang et al. (1995) para a estimativa da Tb de cada cultivar através de seis métodos: desvio padrão em graus-dia $\left(\mathrm{DP}_{\mathrm{gd}}\right)$, desvio padrão em dias $\left(\mathrm{DP}_{\mathrm{d}}\right)$, coeficiente de variação em dias $\left(\mathrm{CV}_{\mathrm{d}}\right)$, coeficiente de variação em grausdia $\left(\mathrm{CV}_{\mathrm{gd}}\right)$, coeficiente de regressão $(\mathrm{CR})$, e X-intercepto ou desenvolvimento relativo (DR).

$\mathrm{O} \mathrm{DP}_{\mathrm{gd}}$ considera a $\mathrm{Tb}$ de cada espécie/cultivar como aquela que resulta no menor desvio padrão em graus-dia (Fagundes et al., 2010), ou seja, no $\mathrm{DP}_{\mathrm{gd}}$ a temperatura basal inferior é selecionada de modo que a variação resultante em graus-dia (GD), usando-se uma série de épocas de transplantios seja mínima (Yang et al., 1995):

$$
D P g d=\sqrt{\frac{\sum_{i=1}^{n}\left(G D_{i}-M G D\right)^{2}}{n-1}}
$$

em que: $\mathrm{DP}_{\mathrm{gd}}=$ desvio padrão em graus-dia; $\mathrm{GD}_{\mathrm{i}}=$ graus-dia acumulados na $i$-ésima época de transplantio utilizando uma série de Tb; MGD = média dos graus-dia acumulados para todas as $i$-ésimas épocas de transplantio; $\mathrm{n}=$ número de épocas de transplantio.

$\mathrm{O}$ método $\mathrm{DP}_{\mathrm{d}}$ baseia-se na premissa de que a $\mathrm{Tb}$ da espécie/cultivar é aquela que resulta no menor desvio padrão entre as diferentes épocas de transplantio (Fagundes et al., 2010) de acordo com (Arnold, 1959):

$$
D P_{d}=\frac{D P_{g d}}{\bar{x}-T b}
$$

em que: $\mathrm{DP}_{\mathrm{d}}=$ desvio padrão em dias; $\mathrm{DP}_{\mathrm{gd}}=$ desvio padrão em graus-dia utilizando uma série de $\mathrm{Tb} ; \bar{x}=$ temperatura média do ar de todas as $i$ épocas $\left({ }^{\circ} \mathrm{C}\right) ; \mathrm{Tb}=$ temperatura basal inferior $\left({ }^{\circ} \mathrm{C}\right)$.

No método do $\mathrm{CV}_{\mathrm{d}}$ a $\mathrm{Tb}$ de cada espécie/cultivar é a que decorre em um menor coeficiente de variação. $\mathrm{O} \mathrm{CV}_{\mathrm{d}}$ é uma relação entre o desvio padrão em dias e o número de dias requerido para atingir determinada fase de desenvolvimento (Yang, et al., 1995) que neste estudo foi representada pela fase de muda:

$$
C V_{d}=\frac{D P_{d}}{\bar{x} d} \cdot 100
$$

em que: $\mathrm{CV}_{\mathrm{d}}=$ coeficiente de variação em dias (\%); $\bar{x} d=$ média do número de dias requerido para atingir determinada fase de desenvolvimento.

$\mathrm{O}$ método do $\mathrm{CV}_{\mathrm{gd}}$ considera o valor de $\mathrm{Tb}$, como aquele que apresentar o menor coeficiente de variação com relação aos graus-dia necessários para atingir determinada fase do desenvolvimento, dado pelo fórmula (adaptado de Yang et al.,1995):

$$
C V_{g d}=\frac{D P_{g d}}{M G D} \cdot 100
$$

O método do CR baseia-se na existência de uma relação linear entre a temperatura média do ar durante a fase de desenvolvimento $\left(\mathrm{Tmed},{ }^{\circ} \mathrm{C}\right.$ ) e os $\mathrm{GD}_{\mathrm{i}}$ necessários para atingir a fase de desenvolvimento considerada (Arnold, 1959):

$$
G D_{i}=a \cdot \text { Tmed }+b
$$

em que: $\mathrm{a}=$ coeficiente angular da regressão linear, $\mathrm{b}=$ coeficiente linear.

$\mathrm{O}$ valor do coeficiente angular da regressão linear simples indica o valor da $\mathrm{Tb}$ correta de cada espécie/cultivar (Fagundes et al., 2010). Valor de 'a' positivo indica que a $\mathrm{Tb}$ usada para calcular a GDi é elevado e maior que o valor correto; valor de 'a' negativo indica $\mathrm{Tb}$ baixa e menor que o valor correto, e valor de 'a' igual zero (ou o mais próximo a zero) indica a $\mathrm{Tb}$ correta (Arnold,1959, Yang et al., 1995).

$\mathrm{O}$ método do $\mathrm{x}$-intercepto ou DR baseia-se na relação linear entre a Tmed durante a fase de desenvolvimento em estudo 
e os valores do DR da espécie/cultivar, utilizando-se a equação (Müller et al., 2009):

$$
D R=a \cdot \text { Tmed }+b, \text { sendo } \mathrm{DR}=100 / \mathrm{N}
$$

em que: $a=$ coeficiente angular da regressão linear, $b=$ coeficiente linear, $100=$ valor arbitrário de ponderação; $\mathrm{N}=$ número de dias de duração da fase de desenvolvimento em cada época de transplantio.

Pelo DR o valor da Tb é a que resulta um desenvolvimento relativo nulo proveniente do prolongamento da regressão linear simples entre o DR da cultivar em função da Tmed (Fagundes et al., 2010). Ou seja, Tb =-b/a (Müller et al., 2009).

Devido a existência de diferença entre os valores de $\mathrm{Tb}$ encontrados em cada método, o valor final da $\mathrm{Tb}$, para cada cultivar de oliveira, foi obtido pela média aritmética dos valores de $\mathrm{Tb}$ encontrados pelos seis métodos.

\section{RESULTADOS E DISCUSSÃO}

O cultivo em diferentes épocas de transplantio no campo proporcionou às mudas de oliveira se desenvolverem em condições meteorológicas distintas durante seu ciclo representado pela fase de muda, as quais foram importantes na estimativa da temperatura basal inferior $(\mathrm{Tb})$ para as duas cultivares (Tabela 1). A média das temperaturas mínimas e máximas durante todo o período de duração do experimento foi, respectivamente, $7,7^{\circ} \mathrm{C}$ e $24^{\circ} \mathrm{C}$. Nesse mesmo período a menor temperatura mínima absoluta do ar foi de $-3,7^{\circ} \mathrm{C}$ (28/06/2011) e a maior temperatura máxima absoluta do ar foi $32^{\circ} \mathrm{C}(01 / 10 / 2011)$.

A diferença entre as condições meteorológicas vigentes durante as três épocas de transplantio também influenciaram na duração da fase de desenvolvimento da muda, apresentando tendência de diminuição da duração com o avanço da época de transplantio. A presença de uma relação inversa entre a temperatura do ar e a duração da fase de muda, indica que quanto menor a temperatura do ar, maior será a duração dessa fase de muda, para as duas cultivares de oliveira. Essa tendência justifica a escolha das três épocas de transplantio (junho, julho e agosto) para a estimativa da $\mathrm{Tb}$. Mesma tendência foi observada em outras culturas como o triticale (Pedro Jr. et al., 2004), a canola (Luz et al., 2012) e para a cultivar de oliveira MGS ASC315 (Lisboa et al., 2012).

Observam-se variações nos valores de $\mathrm{Tb}$ entre os seis métodos (Tabela 2), o que também foi verificado por Barbano et al. (2002) para a ervilha, Andrade (2004) para o milho e o feijão, Pedro Jr. et al. (2004) para o triticale, Lago et al. (2009) para o arroz, Fagundes et al. (2010) para a aspília, e Luz et al. (2012) para a canola. Para a cultivar Grappolo os valores de $\mathrm{Tb}$ variaram entre 6,5 e $11,5^{\circ} \mathrm{C}$, e para a cultivar Maria da Fé variaram entre 6,5 e $7,5^{\circ} \mathrm{C}$

No método do $\mathrm{DP}_{\mathrm{gd}}$ para a cv. Grappolo o valor de $\mathrm{Tb}$ encontrado foi superior a $20^{\circ} \mathrm{C}$ (Figura 1a), sendo considerado um valor não realístico biologicamente (Lago et al., 2009), pois difere consideravelmente dos valores obtidos pelos outros métodos para a mesma cultivar. Para contornar esse problema, considerou-se a $\mathrm{Tb}$ da cv. Grappolo como sendo a média aritmética das temperaturas mínimas dos meses de duração da época de transplantio mais fria, que ocorreu na E3. Dessa forma, o valor considerado como $\mathrm{Tb}$ foi de $6,5^{\circ} \mathrm{C}$.

Os valores de $\mathrm{Tb}$ estimados pelos seis métodos foram semelhantes entre si para a cv. Maria da Fé, sendo ligeiramente superior no $\mathrm{DP}_{\mathrm{gd}}$, semelhante a Barbano et al. (2002), Lago et al. (2009) e Fagundes et al. (2010). Já para a cv. Grappolo os métodos que forneceram os maiores valores de $\mathrm{Tb}$ foram $\mathrm{CR}$ e DR. No entanto, o método do DR obteve uma boa relação linear entre a taxa de desenvolvimento e a temperatura média do ar, com coeficientes significativos e coeficiente de determinação $\left(\mathrm{r}^{2}\right)$ elevado, sendo considerado um excelente método para estimar a Tb (Müller et al., 2009).

Portanto, o valor de $\mathrm{Tb}$ para cada cultivar foi considerado como a média da $\mathrm{Tb}$ obtida pelos seis métodos avaliados. Assim a $\mathrm{Tb}$ para a cv. Grappolo foi de $9,6^{\circ} \mathrm{C}$ e de $6,9^{\circ} \mathrm{C}$ para a cv. Maria da Fé. Esses resultados podem indicar vantagens da cv. Maria da Fé em relação a cv. Grappolo, principalmente em plantios realizados no inverno, onde as temperaturas são amenas. $\mathrm{A} \mathrm{Tb}$ dos vegetais apresenta diferença entre espécies, e entre cultivares dentro da mesma espécie, e pode variar em

Tabela 1 - Caracterização da temperatura do ar durante as épocas de instalação do experimento a campo e duração de cada época de transplantio, correspondente ao período que se estende da data de instalação de cada época até a última observação do número de folha emitida para a fase de muda. Maria da Fé, MG (2011).

\begin{tabular}{cccccc}
\hline Épocas & \multicolumn{2}{c}{$\begin{array}{c}\text { Média da temperatura do ar } \\
\left({ }^{\circ} \mathrm{C}\right)\end{array}$} & \multicolumn{2}{c}{$\begin{array}{c}\text { Duração média da fase de } \\
\text { desenvolvimento (dias) }\end{array}$} \\
\hline & Mínima & Média & Máxima & $\begin{array}{c}\text { cv. } \\
\text { Maria da Fé }\end{array}$ & $\begin{array}{c}\text { cv. } \\
\text { Grappolo }\end{array}$ \\
\cline { 2 - 6 } E1 & 7,0 & 15,4 & 23,7 & 92 & 92 \\
E2 & 7,9 & 16,4 & 24,9 & 92 & 92 \\
E3 & 9,1 & 17,3 & 25,5 & 79 & 65 \\
\hline
\end{tabular}


Tabela 2 - Valores de Tb estimados pelos seis métodos analisados, para as duas cultivares de oliveira (Olea europea L.). Maria da Fé, MG (2011).

\begin{tabular}{ccc}
\hline Métodos & cv. Grappolo & cv. Maria da Fé \\
\hline $\mathrm{DP}_{\mathrm{gd}}$ & $>20^{\circ} \mathrm{C}^{+} / 6,5^{\circ} \mathrm{C}^{*}$ & $7,5^{\circ} \mathrm{C}$ \\
$\mathrm{DP}_{\mathrm{d}}$ & $9,5^{\circ} \mathrm{C}$ & $6,5^{\circ} \mathrm{C}$ \\
$\mathrm{CV}_{\mathrm{d}}$ & $9,5^{\circ} \mathrm{C}$ & $6,5^{\circ} \mathrm{C}$ \\
$\mathrm{CV}_{\mathrm{gd}}$ & $10,5^{\circ} \mathrm{C}$ & $6,5^{\circ} \mathrm{C}$ \\
$\mathrm{CR}$ & $11,5^{\circ} \mathrm{C}$ & $7{ }^{\circ} \mathrm{C}$ \\
$\mathrm{DR}$ & $11^{\circ} \mathrm{C}$ & $7{ }^{\circ} \mathrm{C}$ \\
\hline
\end{tabular}

$\mathrm{DP}_{\mathrm{gd}}=$ método do menor desvio padrão em graus-dia; $\mathrm{DP}_{\mathrm{d}}=$ método do menor desvio padrão em dias; $\mathrm{CV}_{\mathrm{d}}=$ método do coeficiente de variação em dias; $\mathrm{CV}_{\mathrm{gd}}=$ método do coeficiente de variação em graus-dia; $\mathrm{CR}=$ métododo coeficiente de regressão, $\mathrm{DR}=$ método do $\mathrm{x}$-intercepto ou desenvolvimento relativo; ${ }^{+}$Valor não utilizado para calcular a média da Tb (substituído). * Valor obtido pela média aritmética das temperaturas mínimas do ar.
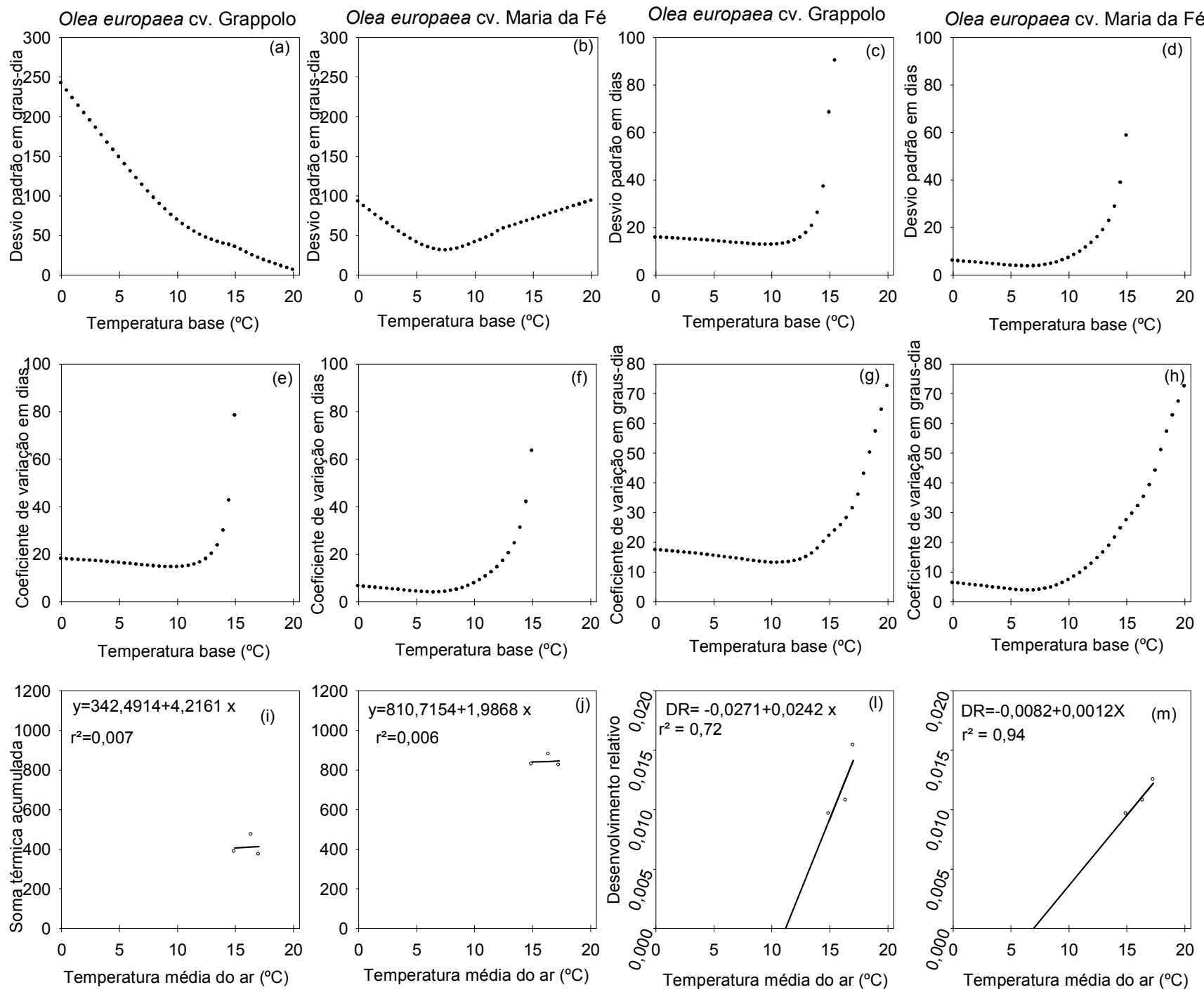

Figura 1 - Representação gráfica dos seis métodos de estimativa da Tb para as cultivares de oliveira Grappolo e Maria da Fé. Maria da Fé, MG, 2011. Paineis a, b método do menor desvio padrão em graus-dia; c,d método do menor desvio padrão em dias; e, f método do coeficiente de variação em dias; $\mathrm{g}$, h método do coeficiente de variação em graus-dia; i,j métododo coeficiente de regressão, 1 , m método do x-intercepto ou desenvolvimento relativo. Os painéis i, j, l, m são representativos dos valores médios de todas as épocas de transplantio. 
função do estádio de desenvolvimento da cultura (Lago et al., 2009). Neste estudo, estimou-se a Tb para o desenvolvimento vegetativo representado pela fase de muda, e os valores de $\mathrm{Tb}$ estimados para a Grappolo $\left(9,6^{\circ} \mathrm{C}\right)$ foram próximos aos valores encontrados para o desenvolvimento vegetativo tanto de culturas perenes, como Eucalyptus grandis $\left(10,0^{\circ} \mathrm{C}\right)$ (Martins et al., 2007), como para algumas culturas anuais, como híbridos de canola $\left(9,9\right.$ e $\left.10^{\circ} \mathrm{C}\right)$ (Luz et al., 2012) e biótipos de arroz

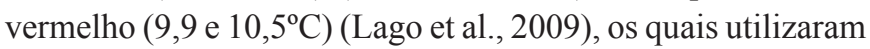
diferentes metodologias de estimativa da Tb. Já para a cv. Maria da Fé $\left(6,9^{\circ} \mathrm{C}\right)$, os valores foram próximos aos encontrados por Chiang e Brown (2007) para diferentes espécies perenes como Quercus velutina (carvalho negro) e Acer saccharum $\left(5^{\circ} \mathrm{C}\right)$, Acer rubrum (acer vermelho) e Betula popifolia (Bétula cinzenta) $\left(7,5^{\circ} \mathrm{C}\right)$; por Lucas et al. (2012) para melancia $\left(7,0^{\circ} \mathrm{C}\right)$; por Müller et.al. (2009) para azevém $\left(7,0\right.$ à $\left.8,0^{\circ} \mathrm{C}\right)$; por Angus et al. (1981) para cártamo $\left(7,4^{\circ} \mathrm{C}\right)$; e por Martins et al. (2007) para Eucalyptus saligna $\left(8^{\circ} \mathrm{C}\right)$.

Cabe ressaltar que esses valores estimados para as duas cultivares são próximos, porém mais baixos (principalmente para a cv. Maria da Fé) do que os valores encontrados na literatura para oliveira. Por exemplo, Martins et al. (2012) obtiveram valores de $\mathrm{Tb}$ de 10,5 e $11^{\circ} \mathrm{C}$ para as cultivares de oliveira Arbequina e MGS ASC315 utilizando uma metodologia distinta das utilizadas neste estudo, e Alcalá e Barranco (1992) entre 10 e $13^{\circ} \mathrm{C}$, para o florescimento de diferentes cultivares de oliveira na Espanha. Outros autores como Melo-Abreu et al. (2004) encontraram $\mathrm{Tb}$ de 8,5 e $8,8^{\circ} \mathrm{C}$ para o processo de quebra de dormência; e Ayerza e Sibbett (2001) encontraram valores entre 0 e $4,5^{\circ} \mathrm{C}$ para o processo de vernalização em várias cultivares de oliveira no Texas, e em alguns lugares da Argentina, Espanha, Itália, Peru e México. Informações básicas sobre o desenvolvimento vegetativo, como a estimativa da $\mathrm{Tb}$, em cultivares de oliveira são importantes para conhecer as cultivares mais adaptadas às condições climáticas de cultivo, e as necessidades da cultura em condições meteorológicas distintas do seu centro de origem (Martins et al., 2012).

Certamente, esse tipo de informação é fundamental para o planejamento, manejo e tratos culturais da oliveira em condições de campo.

\section{CONCLUSÕES}

A temperatura basal inferior $(\mathrm{Tb})$ para as duas cultivares de oliveira variou entre os seis métodos de cálculo analisados neste estudo. A Tb para o desenvolvimento vegetativo, representado pela fase de muda, foi de $9,6^{\circ} \mathrm{C}$ para a cultivar Grappolo e $6,9^{\circ} \mathrm{C}$ para a cultivar Maria da Fé, demonstrando que a última é mais tolerante à temperaturas mais baixas e mais exigente em frio.

\section{AGRADECIMENTOS}

Ao Conselho Nacional de Desenvolvimento Científico e Tecnológico (CNPq) pelo apoio financeiro e auxílio concedido ao primeiro autor. À Fundação de Amparo à Pesquisa do Estado de Minas Gerais (FAPEMIG), referente ao projeto APQ-0139213, pelo apoio financeiro. À Empresa de Pesquisa Agropecuária de Minas Gerais (EPAMIG) por toda a infraestrutura disponibilizada.

\section{REFERÊNCIAS BIBLIOGRÁFICAS}

ALCALÁ, A.R.; BARRANCO, D. Prediction of flowering time in olive for the Cordoba Olive Collection. Horticultural Science, v. 27, n. 11, p. 1205-1207, 1992.

ANDRADE, R. G. Estimativa da temperatura base inferior e avaliação dos modelos beta, RCM e GDD em diferentes subperíodos das culturas de milho, arroz e feijão. 2004. 67f. Dissertação (Mestrado em Meteorologia Agrícola) Universidade Federal de Viçosa, Viçosa, 2004.

ANGUS, J.F.; CUNNINGHAM, R.B.; MONCUR, M.W.; MACKENZIE, D.H. Phasic development in field crops. I. Thermal response in the seedling phase. Field Crops Research, v. 3, p. 365-378, 1981.

ARNOLD, C. Y. The Determination and Significance of the Base Temperature in a Linear Heat Unit System. American Society for Horticulture Science, v. 74, p. 430-445, 1959. ARNOLD, C. Y. Maximum-Minimum Temperature as a Basis for Computing Heat Units. American Society for Horticulture Science, v. 76, p. 682-692, 1960.

ASSUMPÇÃO NETO, A. Plastocrono e filocrono aparentes anual em Araucaria angustifolia (BERT.) O. KTZE., no município de Colombo - PR. 2008. 55f. Dissertação (Mestrado em Agronomia) - Programa de Pós-graduação em Agronomia, Universidade Federal do Paraná, 2008.

AYERZA, R.; SIBBETT, G.S. Thermal adaptability of olive (Olea europaea L.) to the Arid Chaco of Argentina. Agricultural, ecosystems and Environment, v. 84, n. 3, p. 277-285, 2001.

BARBANO, M.T.; WUTKE, E.B.; BRUNINI, O.; AMBROSANO, E.J; CASTRO, J.L.de.; GALLO, P.B.; PEREIRA, J.C.V.N.A.; MARTINS, A.L.M. Temperaturabase e soma térmica para cultivares de ervilha (Pisum sativum L.) Revista Brasileira de Agrometeorologia, ,v. 10, n. 1, p. 75-82, 2002.

CHIANG, J.M; BROWN, K.J. Improving the budburst phenology subroutine in the forest carbon model PnET. Ecological Modelling, v. 205, p. 515-526, 2007.

FAGUNDES, J.D.; STRECK, N.A.; STORCK, L.; REINIGER, L.R. Temperatura base e soma térmica de subperíodos de 
desenvolvimento de Aspilia montevidensis. Bragantia, v. 69, n. 9, p. 499-507, 2010.

LAGO, I.; STRECK, N.A.; CARVALHO, M.P.; FAGUNDES, L.K.; PAULA, G.M.de.; LOPES, S.J. Estimativa da temperatura base do subperíodo emergência-diferenciação da panícula em arroz cultivado e arroz vermelho. Revista Ceres, v. 56, p. 288-295, 2009.

LIMA, E.P.; SILVA, E.L.da. Temperatura base, coeficientes de cultura e graus-dia para cafeeiro arábica em fase de implantação. Revista Brasileira de Engenharia Agrícola e Ambiental, v. 12, n. 3, p. 266-273, 2008.

LISBOA, P.M.M.; MARTINS, F.B.; ALVARENGA, M.I.N.; VIEIRA NETO, J.; REIS, D.F.da. Desenvolvimento vegetativo de duas cultivares de oliveira. Ciência Rural, v. 42, n. 9, p. 1556-1562, 2012.

LOZADA, B.I.; ANGELOCCI, L.R. Determinação da temperatura-base e graus-dia para a estimativa da duração do sub-período de semeadura à floração de um híbrido de milho. Revista Brasileira de Agrometeorologia, v. 7, n. 1, p. 31-36, 1999.

LUCAS, D.D.P.; STRECK, N.A.; BORTOLUZZI, M.P.; TRENTIN, R.; MALDANER, I. Temperatura base para emissão de nós e plastocrono de plantas de melancia. Revista Ciência Agronômica, v. 42, p. 288-292, 2012.

LUZ, G.L.da; MEDEIROS, S.L.P.; TOMM, G.O.; BIALOZOR, A.; AMARAL, A.D.do.; PIVOTO, D. Temperatura base inferior e ciclo de híbridos de canola. Ciência Rural, v. 42, n. 9, 2012.

MARTINS, F.B.; CORDEIRO, J.C.da.; STRECK, N.A. Estimativa da temperatura-base para emissão de folhas e do filocrono em duas espécies de eucalipto na fase de muda. Revista Árvore, v. 31, n. 3, p. 373-381, 2007.

MARTINS, F.B.; REIS, D.F.da.; PINHEIRO, M.V.M. Temperatura base e filocrono em duas cultivares de oliveira. Ciência Rural, v. 42, n. 11, p. 1975-1981, 2012.

MARTINS, F.B.; STRECK, N.A. Aparecimento de folhas em mudas de eucalipto estimado por dois modelos. Pesquisa Agropecuária Brasileira, v. 42, n. 8, p. 1091-1100, 2007.
MELO-ABREU, J.P., BARRANCO D., CORDEIRO A.M., TOUS J., ROGADO B.M., VILLALOBOS F.J. Modelling olive flowering date using chilling for dormancy release and thermal time, Agricultural and Forest Meteorology, v. 125, p. 117-127, 2004.

MÜLLER, L.; MANFRON, P.A.; MEDEIROS, S.L.P.; STRECK, N.A.; MITTELMMAN, A.; NETO, D.D.; BANDEIRA, A.H.; MORAIS, K.P. Temperatura base inferior e estacionalidade de produção de genótipos diploides e tetraploides de azevém. Ciência Rural, v. 39, n. 5, p. 1343-1348, 2009.

PEDRO Jr., M.J.; CAMARGO, M.B.P.de; MORAES, A.V.C.de.; FELÍCIO, J.C.; CASTRO, J.L.de. Temperatura-base, grausdia e duração do ciclo para culturas de triticale. Bragantia, v. 63, n. 3, p. 447-453, 2004.

ROSA, H.T.; WALTER, L.C.; STRECK, N.A.; ALBERTO, C.M. Métodos de soma térmica e datas de semeadura na determinação de filocrono de cultivares de trigo. Pesquisa Agropecuária Brasileira, v. 44, n. 11, p. 1374-1382, 2009. SANZ-CORTÉS, F.; MARTINEZ-CALVO, J.; BADENES, M. L.; BLEIHOLDER, H.; HACK, H.; LLACER, G.; MEIER, U. Phenological growth stages of olive trees (Olea europaea L.). Annals of Applied Biology, v. 140, n. 2, p. 151-157, 2002.

SINCLAIR, T.R.; GILBERT, R. A.; PERDOMO, R. E.; SHINE Jr., J. M.; POWELL, G.; MONTES, G. Sugarcane leaf area development under field conditions in Florida, USA. Field Crops Research, v.88, n.1, p.171-178, 2004.

VIEIRA NETO, J.; OLIVEIRA, A. F.; CAPRONI, C. M.; VILLA, F.; SILVA, L. F. O. Desempenho de jardins clonais de oliveira (Olea europaea L.) em cortes sucessivos visando a sua propagação por estaquia. Cerne, v. 17, n. 1, p. 117122, 2011.

YANG S.; LOGAN J.; COFFEY D.L. Mathematical formulae for calculating the base temperature for growing degree days. Agricultural and Forest Meteorology, v. 75, p. 61-74, 1995. 